\section{Explaining Formation of Astronomical Jets Using Dynamic Universe Model}

Satyavarapu Naga Parameswara Gupta*

Bhilai Steel Plant, Bhilai, India

\begin{abstract}
Astronomical jets are observed from the centres of many Galaxies including our own Milkyway. The formation of such jet is explained using SITA simulations of dynamic universe model. For this purpose the path traced by a test neutron is calculated and depicted using a set up of one densemass of the mass equivalent to mass of Galaxy center, 90 stars with similar masses of stars near Galaxy center, mass equivalents of 23 Globular Cluster groups, 16 Milkyway parts, Andromeda and Triangulum Galaxies at appropriate distances. Five different kinds of theoretical simulations gave positive results. The path travelled by this test neutron was found to be an astronomical jet emerging from Galaxy center.
\end{abstract}

Keywords: Astronomical jets; Dynamic universe model; SITA calculations

\section{Introduction}

Dynamic Universe model is a singularity free tensor based math model. The tensors used are linear without using any differential or integral equations, there will not be any divided by zero errors, multiple real solutions or any other imaginary solutions for a single set of input data. Only one calculated output set of values exists. Data means properties of each mass like its three dimensional coordinates, velocities, accelerations and it's mass. In this paper, the set of Galaxy center of the Milkyway along with ninety numbers Wolf-Rayet stars is simulated. This whole set is under the continuous and dynamical influence of twenty three globular cluster groups, sixteen Milkyway parts, Andromeda and Triangulum Galaxies. It is a total of 133 masses in this work. This galactic center can be of any another Galaxy also but the outer parts will vary. The dynamical gravitation effect of all this set of masses on a test neutron is calculated and depicted as graphs. Various cases of simulations and their output graphs are discussed in output section. For any N-body problem calculations, the more accurate our input data the better will the calculated results; one should take extreme care, while collecting the input data.

Basic constituent parts of this paper are input data collection, procedure for calculations, various simulations and their output graphs, and lastly discussions/conclusions. In the 'Procedure for calculations' section a discussion can be seen about the total time taken for calculations and number of iterations in each type of simulations.

\section{Input data collection}

For conducting these simulations/calculations using a N-body problem solution called dynamic universe model is used. The required real observational data is collected from various sources. That included many research papers and web-pages. All these are referenced. Even Wikipedia also was checked many times for better understanding of some of the practices involved.

\section{Reference to coordinate system}

Sun is the reference as usual with it's mass as $1.99 \mathrm{E}+30 \mathrm{~kg}$, and the Helio Centric Ecliptic Xyz coordinate values of Sun as on 01.01.2000@00.00:00 hrs are Xecliptic=0.0, yecliptic=0.0 and Zecliptic $=0.0$ Meters. Even though SUN is the reference it will not be used in the calculations as Sun is very far off from Galaxy center.
The stars S1 to S100 will be staying in nearby locations to Galaxy (MILKY Way) center and they will be used in calculations. There is no Coordinate system which is centered on Milkyway center, which would have been a better choice for doing these calculations.

\section{Galactic center}

The distance of Galactic Center is of the order of 26000 light-years from solar system. Its direction is having a Right Ascension of $17 \mathrm{~h} 45$ $\mathrm{m} 40.045 \mathrm{~s}$ and Declination of (-) $29^{\circ} 0^{\prime} 27.9^{\prime \prime}$. Its mass can be estimated as 4.1 million $M_{\odot}$ or about $8.2 \times 10^{36} \mathrm{~kg}$. The orbits of WR star S14 [1], specify that the radius is no more than 6.25 light-hours. In another paper its mass is of the order of 4.31 million $M_{\odot}$ [2]. Lower value of both the estimates is taken here. The author needs to say one more thing at this point, about the super massive Blackhole [3] at the center of Milkyway according to BBC news. He could not find any further published paper about it on internet. A densemass is selected instead of Black hole. This paper requires only mass and coordinates of this densemass, size is not important for these calculations.

\section{Wolf-Rayet stars (WR stars or Galaxy Center Stars)}

Masses of WR stars: Masses of WR stars near Galaxy core are named like S1 to S100 etc. Their masses are simulated using the Excel formula

$$
=1.99 \mathrm{E}+30 \times(\text { RAND }() \times(24-16)+16)
$$

This formula generates random numbers between 24 and 16. This generated random number is multiplied with solar mass of $1.99 \mathrm{e} 30 \mathrm{~kg}$ to give a star mass which is minimum 16 times to 24 times solar mass [4].

Three dimensional coordinates: Three dimensional coordinates of

*Corresponding author: Satyavarapu Naga Parameswara Gupta, Retired Assistant General Manager, Bhilai Steel Plant, Bhilai, India, Tel: 919407980419; E-mail: snp.gupta@gmail.com

Received June 17, 2015; Accepted July 28, 2015; Published August 07, 2015

Citation: Gupta SNP (2015) Explaining Formation of Astronomical Jets Using Dynamic Universe Model. J Astrophys Aerospace Technol 3: 119. doi:10.4172/2329-6542.1000119

Copyright: (c) 2015 Gupta SNP. This is an open-access article distributed under the terms of the Creative Commons Attribution License, which permits unrestricted use, distribution, and reproduction in any medium, provided the original author and source are credited. 
Citation: Gupta SNP (2015) Explaining Formation of Astronomical Jets Using Dynamic Universe Model. J Astrophys Aerospace Technol 3: 119. doi:10.4172/2329-6542.1000119

these WR-Stars are simulated using the following Excel formula.

- For $\mathrm{x}$ ecliptic the formula applied $=\$ \mathrm{~K} \$ 31 \times(\mathrm{R}$ AND ()$\times$ $(0.00000478802)+1)$

- For y ecliptic the formula applied $=\$ \mathrm{~L} \$ 31 \times(\mathrm{R}$ AND ()$\times$ $(0.00000478802)+1)$

- For $\mathrm{z}$ ecliptic the formula applied $=\$ \mathrm{M} \$ 31 \times(\mathrm{R}$ AND ()$\times$ $(0.00000478802)+1)$

Distances: Distances of the above simulated stars are calculated using above simulated coordinates:

- For the Distances the Excel formula used

$=\left((\mathrm{K} 32)^{\wedge} 2+(\mathrm{L} 32)^{\wedge} 2+(\mathrm{M} 32)^{\wedge} 2\right)^{\wedge} 0.5$

\section{Test Particle... Neutron}

Initial position and mass of the test particle Neutron: It was chosen as it is electrically neutral. The mass of this was taken from Wikipedia. Position of Neutron was taken near the first Galaxy center star. A value of 1000000000 meters for $\mathrm{x}, 2000000000$ for $\mathrm{y}$ and for $\mathrm{z}$ 3000000000 meters were added to the coordinates of first star and these values were taken as initial $\mathrm{x}, \mathrm{y}, \mathrm{z}$ positions for the Neutron.

Initial velocity for the Neutron: An initial velocity of $1 \%$ of velocity of light was taken. That is $\mathrm{V}=30000 \mathrm{~km} / \mathrm{s}=30000000 \mathrm{~m} / \mathrm{sec}$.

Direction: Direction of test Neutron is taken towards Milky Way center. The direction cosines are calculated using the formula for equation of line through point $x_{0}, y_{0}, z_{0}$ and $x_{1}, y_{1}, z_{1}$ :

$$
\frac{x-x_{0}}{x_{1}-x_{0}}=\frac{y-y_{0}}{y_{1}-y_{0}}=\frac{z-z_{0}}{z_{1}-z_{0}}
$$

Initial calculated velocities: The calculated XYZ velocities for the Neutron directed from position of Neutron to Galaxy center are as xeclipticv $=(-)$ 3363.96588, yeclipticv $=(-) 25846.79996$ and zeclipticv $=(-) 14853.50684 \mathrm{M} / \mathrm{Sec}$

\section{Time-step}

Time-step is the elapsed time between iterations. Sometimes a time-step of 1 second is given initially to stabilize the system for two iterations. A time step of 100 years was taken in some of these calculations.

\section{Initial conditions}

The initial conditions like Mass and Initial distance from Sun in $\mathrm{x}$ direction, are shown in Figures 1 and 2. The spike at Mass no.132 indicates Andromeda Galaxy. In Figure 3 the mass distribution taken here for the WR stars is shown.The graphs are provided for visual understanding of the initial conditions for doing these simulations.

\section{Starting conditions used in various files}

Starting conditions of Various files used in this paper are given below. Neutron mass and all the other distances were not changed, they were maintained the same in all these theoretical experiments as discussed above.

First file: ' Vvtc EUREKA PERP rev Results 2002xGC Less NS $2 \mathrm{sec}$ Densemass.xls' or 'Vvtc rev Results 2002xGC Less NS $2 \mathrm{sec}$ Densemass.xls'

Here in this case GC mass is $8.20 \mathrm{E}+38 \mathrm{~kg}$ near star masses are in the range $3.57 \mathrm{E}+30 \mathrm{~kg}$. Neutron initial xyz velocities are $3359.4 \mathrm{~m} / \mathrm{s}$,

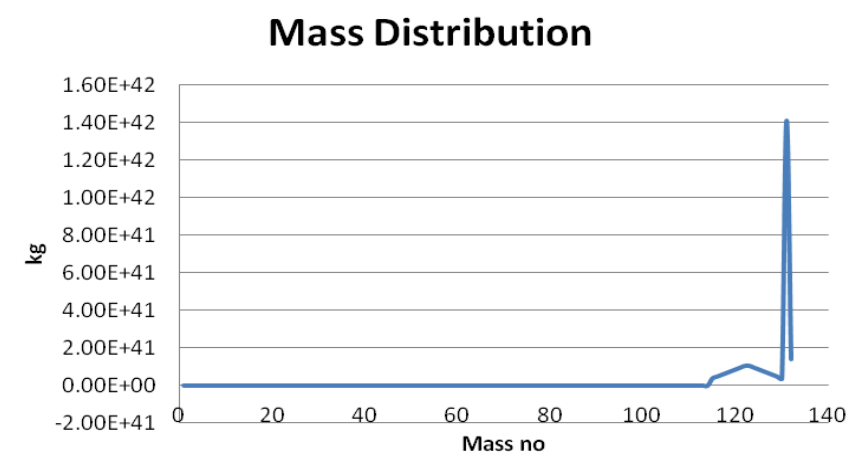

Figure 1: All the masses (Units $\mathrm{kg}$ ) in this simulation are shown here. The spike is Andromeda Galaxy.

\section{Initial Distance from Sun}

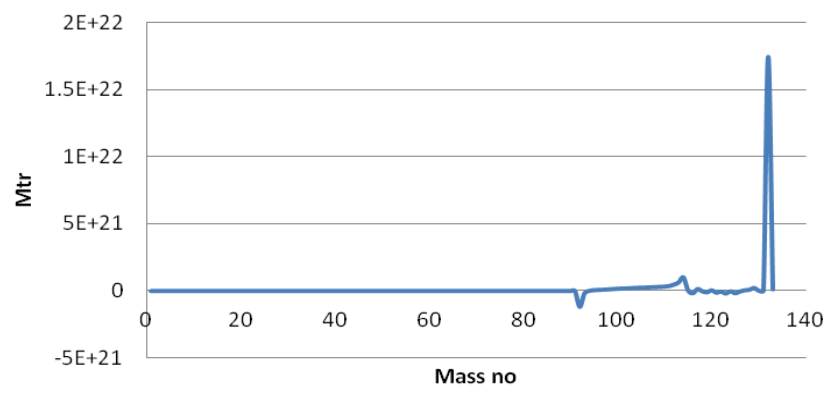

Figure 2: The ecliptic $X$ coordinate distances of the mass from Sun in meters is representedin is shown here.

\section{Mass distribution of WR stars}

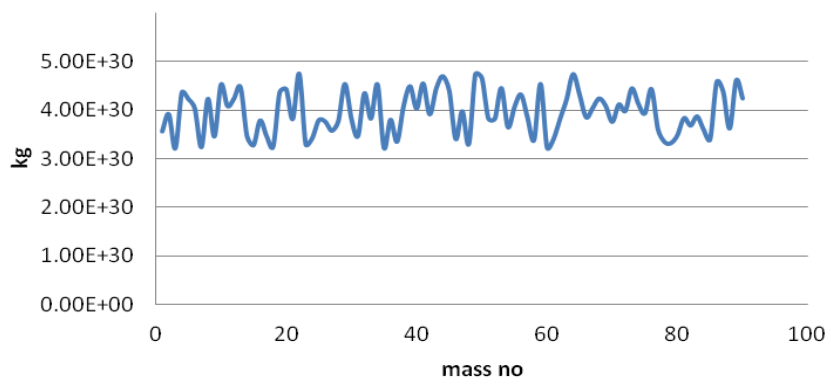

Figure 3: Shows the masses of Wolf-Rayet stars (WR stars or Galaxy Center Stars) used in this simulation.

$25837.6 \mathrm{~m} / \mathrm{s}$, and $14839.8 \mathrm{~m} / \mathrm{s}$, time step=1 sec.

\section{Second file: "Vvtc rev 2000 100xGC Less NS Densemass.xls"}

Here in this case GC mass is $8.20 \mathrm{E}+38 \mathrm{~kg}$ near star masses are in the range $3.57 \mathrm{E}+30 \mathrm{~kg}$. Neutron initial xyz velocities are $3363.9 \mathrm{~m} / \mathrm{s}$, $25846.8 \mathrm{~m} / \mathrm{s}$, and $14853.5 \mathrm{~m} / \mathrm{s}$, time step=1 sec.

\section{Third file: "Vvtc rev xy BENT 2000 100xGC Less NS Densemass.xls"}

Here in this case GC mass is $8.20 \mathrm{E}+38 \mathrm{~kg}$ near star masses are in the range $3.57 \mathrm{E}+30 \mathrm{~kg}$. Neutron initial xyz velocities are $3363.9 \mathrm{~m} / \mathrm{s}$, $25846.8 \mathrm{~m} / \mathrm{s}$, and $14853.5 \mathrm{~m} / \mathrm{s}$, time step=1 sec. 


\section{Fourth file: "Vvtc rev 2000 Galaxy core Less Densemass.xls"}

Here in this case GC mass is $8.20 \mathrm{E}+36 \mathrm{~kg}$ near star masses are in the range $3.57 \mathrm{E}+30 \mathrm{~kg}$. Neutron initial xyz velocities are $3363.9 \mathrm{~m} / \mathrm{s}$, $25846.8 \mathrm{~m} / \mathrm{s}$, and $14853.5 \mathrm{~m} / \mathrm{s}$, time step=1 sec.

\section{Mathematical Background}

The mathematics of Dynamic Universe is available in many published papers. The following Equation (7) is the basis for many calculations. This basic equation is sufficient for almost all practical purposes

$$
\Phi_{e x t}(\alpha)=-\sum_{\substack{\beta=1 \\ \alpha \neq \beta}}^{N^{\gamma}} \frac{G m_{\beta}^{\gamma}}{\left|x^{\gamma \beta}-x^{\gamma \alpha}\right|}-\sum_{\substack{\beta=1 \\ \alpha \neq \beta}}^{N^{\delta \gamma}} \frac{G m_{\beta}^{\delta \gamma}}{x^{\delta \gamma \beta}-x^{\delta \gamma \alpha} \mid}
$$

This concept can be extended to still higher levels in a similar way.

\section{SITA (Simulation of Inter-Intra-Galaxy Tautness and Attraction forces)}

SITA is a totally non-general relativistic algorithm. Here in NO way GR effects are taken into consideration. No space-time continuum. No $\lambda$ factor to introduce repulsion between Galaxies at any distance. In this SITA Simulation Universe is assumed to be dynamically moving and rotating. This is not a static model as assumed by Newton. Additionally on SITA, a inhomogeneous and anisotropic lumpy universe was assumed.

\section{Procedure}

The quest is simple. We need to find out on which input conditions the path traced by neutron will bend perpendicularly. Disappointingly high different numbers of varieties of theoritical experiments with different input conditions were conducted. Using the above defined initial values the SITA calculations procedure gave the data outputs. A large amount of output data was produced. Here different input conditions means different neutron velocities ranging from full light velocity to $1 \%$ of velocity of light in different directions. This output data was analysed using graphs and checked results. Mistakes were corrected. Finally we could get results which were thought probably never possible. All the experiments and outputs were logged. Only final cases are discussed in the next section.

Another hurdle faced is the laptop getting heated up. A new computer has to be purchased with a forced cooling fan. For a simple retired person it is a difficult job to spare some money. But any way, each experiment lasted for a duration of 2 to 6 hours of rigorous calculations by the new computer. Power failures combined with UPS (uninterrupted power supply unit) failures caused hevock many times. Everything needed to be restarted

\section{Results from Simulations}

\section{Abbreviations}

In most of the graphs in this document, we will use GC for Galaxy center or Milkyway center, NS for near star or a Star moving close to GC, PM: point mass or mass, the equivalent mass of the body situated at its gravitational center. Pos means the ecliptic Cartesian xyz coordinate position of the point mass or Neutron, and sx, sy and sz denote positions of xyz axes. Unit of all distances is meters. Vel means the ecliptic xyz velocities associated with the point mass at that instant of time and position and vx, vy and vz denote velocities of point mass in xyz axes. Unit of all velocities is meters/second. Another word rev is used for revised, bend is for bending, itr is used for iteration number and perp is used for perpendicular.

Results consolidation: The details of various final values for the different theoretical experiments are consolidated in the file: ... "vvtc Eureka perp results consolidation.xls".

Positive bending results: Using the input data and starting conditions as discussed in the previous section, many theoretical experiments were conducted. Finally there is a ray of hope. The first moon is visible. The galaxy Densemass astronomical jets emerging from the Milkyway centre is coming true. There was a perpendicular movement observed in a xy pos movement graph. (sy vs sx position).

These observations are stored in the attached file "Vvtc EUREKA PERP rev Results 2002xGC Less NS 2 sec Densemass.xls"

The observation is shown in the Figure 4. Power failures happened many times during running of these files, it is sometimes almost after 6 hours of after starting. Because of the power failures I reduced number of iterations per run in the file "Vvtc rev Results 100xGC Less NS 2 sec Densemass.xls". This File was opened and changed the nuimber of iterations to 1000 from 2000 and then it ran for 5 hours each twice. Later same practice was continued for all the other files.

Positive bending results from the file "Vvtc rev 2000 100xGC Less NS Densemass.xls" are shown in Figure 5. Neutron path bending is visible in the plots of XY postions of neutron only. But not in all the other graphs like where $\mathrm{z}$-axis is present (Figure 6).

\section{Eureka Nuetron Pos Gone perp at GC}

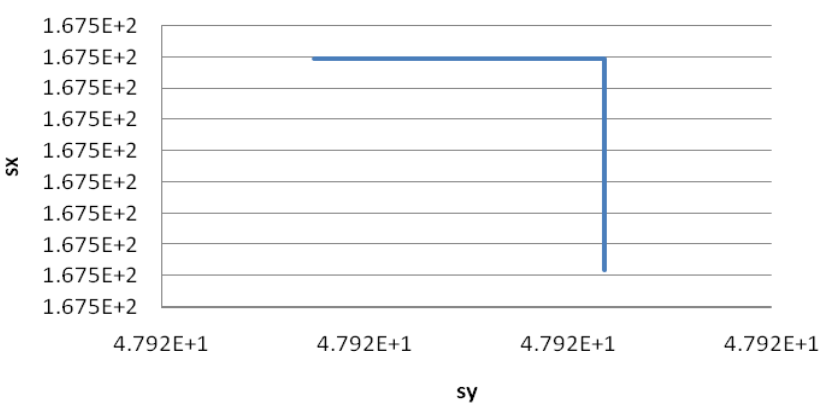

Figure 4: A sx-sy graph showing sudden bending of Neutron path at Galaxy Center.

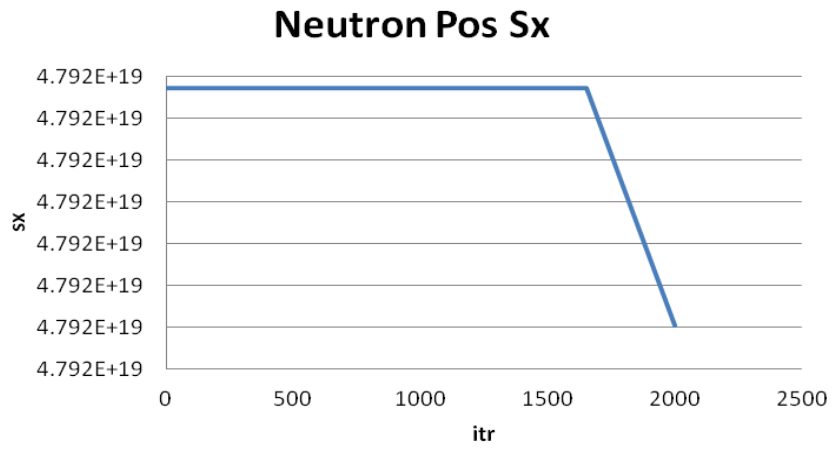

Figure 5: A sx vs itr graph showing sudden bending of Neutron path at Galaxy Centeron the iteration 1653. 


\section{Neutron Pos sx--Bent at Itr 1653}

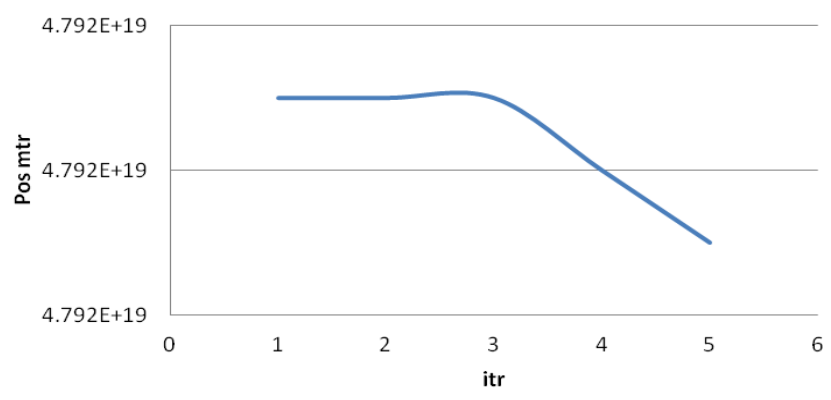

Figure 6: Sx position vs iteration graph in the vicinity of iteration 1653 showing bending.

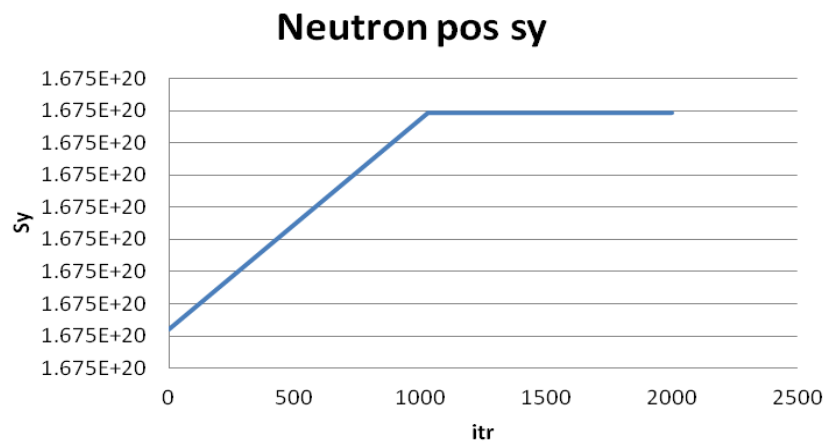

Figure 7: Position of SY bending during iteration 1031.

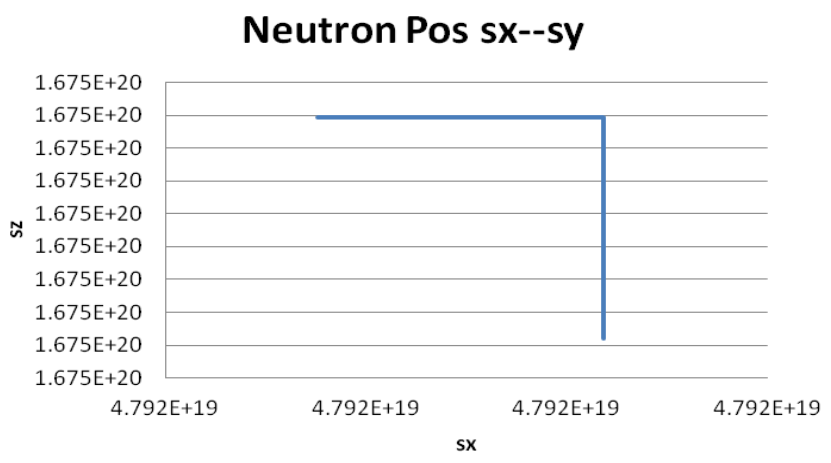

Figure 8: Sx vs sy graph showing bending.

Positive bending results from the files: These files also produced similar graphs "Vvtc rev 2000 Galaxy core Less Densemass.xls" and "Vvtc rev xy BENT 2000 100xGC Less NS Densemass.xls"

Bend is visible in the Figures 7-11.

Bending in neutron $\mathrm{z}$-axis (sz) position path is not visible in all the iterations (Figures 12-14).

\section{Discussion}

\section{Introduction to dynamic universe model}

Dynamic universe model of cosmology uses tensors without differential and integral equations, and gives unique solutions. SITA is the name of one method of programming used for calculation of tensor evaluations in dynamic universe model. Tensors are generally tough to understand interpret and appreciate. This is mainly because it is the number of equations that each tensor that will be subdivided into. The overall concept is difficult to comprehend.

Now let us see what dynamic universe model of cosmology is... It uses tensor mathematics based on Newtonian physics. This mathematics used here is simple and straightforward. All the mathematics and the Excel based software details are explained in the three books published by the author [5-7]. In the first book, the solution to $\mathrm{N}$-body problem-called dynamic universe model (SITA) is presented; which is singularity-free, inter-body collision free and

\section{Neutron pos sy--bend at 1031}

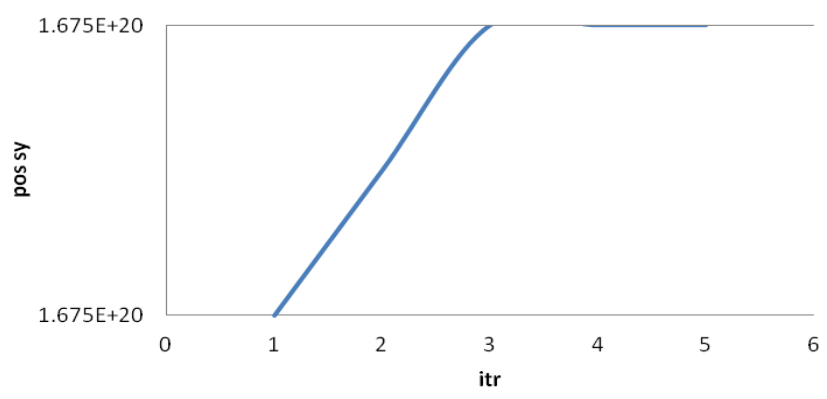

Figure 9: Detailed bending of sy at the iteration 1031 in position.

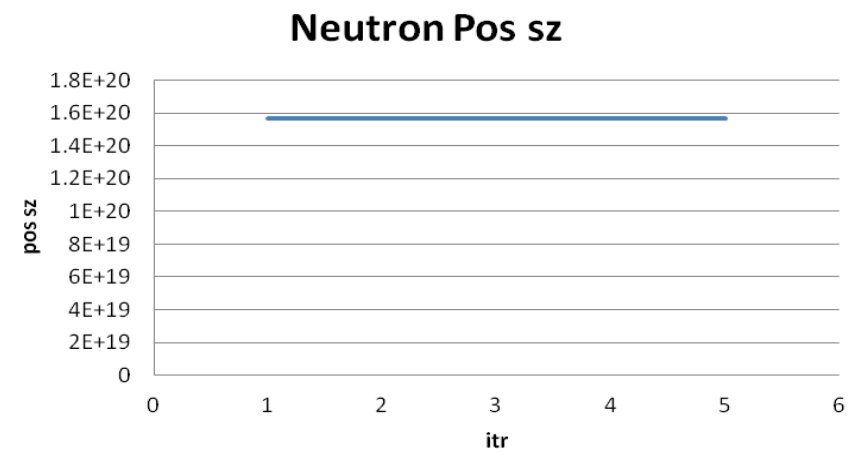

Figure 10: Detail of neutron position of SZ during vicinity of iteration 1031. This is clear from graph, the path of neutrons is not bending in the sz direction.

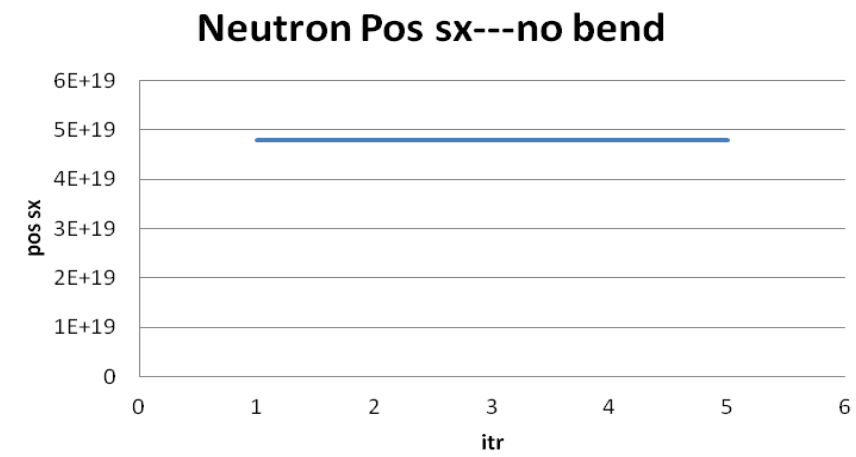

Figure 11: Detail of neutron position of Sx during vicinity of iteration 1031. This is clear from graph, the path of neutrons is not bending in the sx direction. 


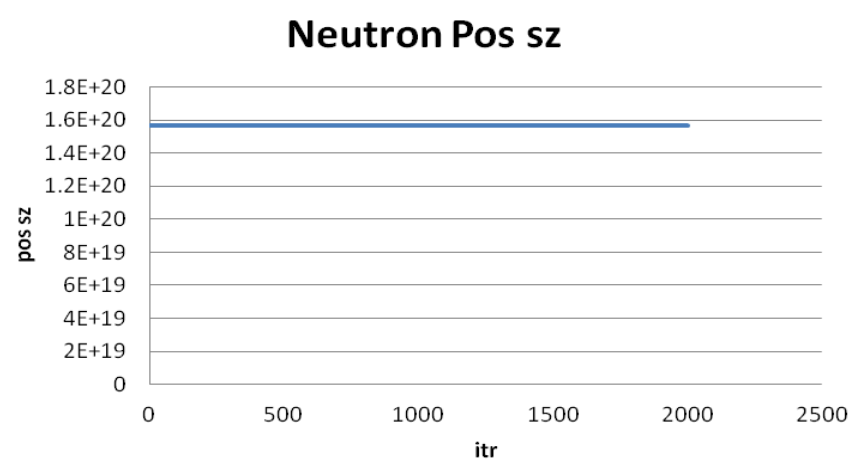

Figure 12: SZ Position path of neutron during all the iterations. This is clear from graph, the path of neutrons is not bending in the $S z$ direction during the whole 2000 iterations. We detailed the SZ during 1031 to see if any bending exists in Figure 10

\section{Neutron Pos sz}

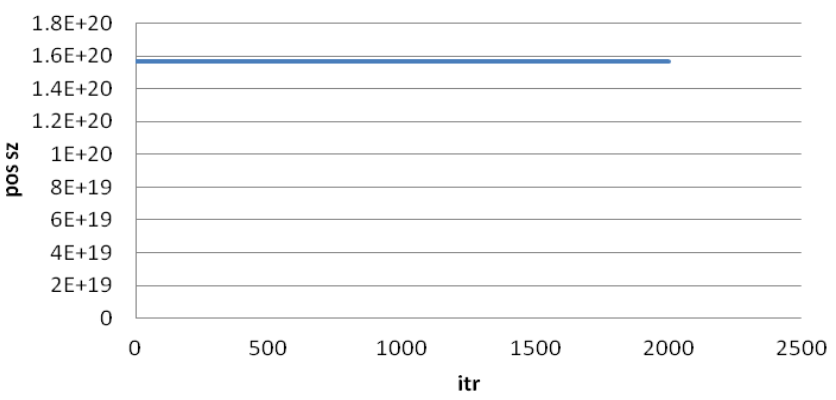

Figure 13: SX Position path of neutron during all the iterations. This is clea from graph, the path of neutrons is not bending in the SX direction during the whole 2000 iterations. We detailed the SX during 1031 to see if any bending exists in Figure 11

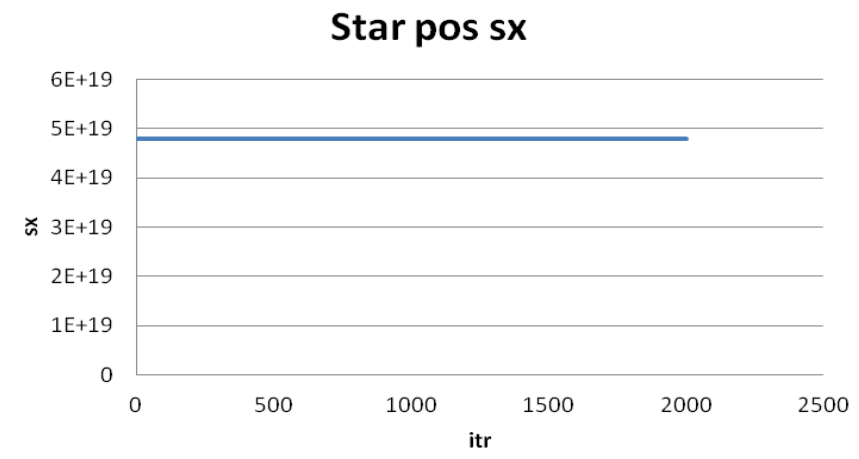

Figure 14: SX Position path of a Star during all the iterations. This is clear from graph, the path of neutrons is not bending in the SX direction during the whole 2000 iterations.

dynamically stable. This is the basic theory of dynamic universe model published in 2010 [5]. The second book in the series describes the equations and SITA software in EXCEL emphasizing the singularity free portions. It explains more than 21,000 different equations [6]. The third book describes the SITA software in EXCEL in the accompanying CD/DVD emphasizing mainly HANDS ON usage of a simplified version in an easy way. The third book contains explanation for 3000 equations instead of earlier 21,000 [7]. With this same SITA setup, many physical problems were solved, which are otherwise not possible. For using this SITA, we have to give the initial values of masses and cartesian X Y Z "coordinates of positions, velocities, and accelerations". Feeding accelerations is not compulsory. Velocities are also not very important, after little iteration of calculations, all the three dimensional Velocities and accelerations will be formed automatically. A point to be noted here is that the dynamic universe model never reduces to general relativity on any condition. The fourth book $[8,9]$ in the series on dynamic universe model: SITA, gave simulations that predicted the existence of the large number of blue-shifted galaxies in 2004, ie., more than about 35 40 blue-shifted galaxies known at the time of Astronomer Edwin Hubble in 1930s. The far greater numbers of blueshifted galaxies was confirmed by the Hubble space telescope (HST) observations in the year 2009.

This dynamic universe model can be used for solving general $\mathrm{N}$-body problem. This method solved many unsolved problems earlier like galaxy disk formations, missing mass in galaxies, pioneer anomaly, non-collapsing large scale mass structures, new horizons trajectory predictions etc. We will start with Equation 25, which is a tensor equation, which will be devided into 21000 linear equations on which SITA calculations work. This is the main equation giving many results, that are not possible otherwise today. Now it attempts to solve new problems like

a. Variable mass rocket trajectory problem.

b. Explaining very long baseline interferometry (VLBI) observations[10].

c. Astronomical jets observed from Milkyway Center.

d. Dynamic universe model's prediction "no dark matter" in the universe came true! [11].

To support dynamic universe model the we can find the following supporting observations.

a. Mathematical prediction of existence of blue shifted galaxies came true.

b. SN1987A-Neutrino emission.

c. The first redshifted Quasar 3C273 is blue shifted (supporting existence of blue shifted galaxies).

d. The most distant Quasar eso1122 found to have a blue Shift of 0.110473 (supporting existence of blue shifted galaxies).

\section{Solving new problems}

Variable mass rocket trajectory problem: When the rocket is moving due to thrust of the fuel in it, its mass will continuously reduce. The positions and gravitation forces of Moon, Sun, Earth and other planets will dynamically change. Dynamic Universe Model predicts such trajectory continuously. This will be very useful for optimizing the overall mission efficiency for reducing the thruster rocket sizes and increasing payload capacity of rocket.

Explaining large variation in the gravitational bending results of VLBI: In this, the effect of universal gravitational force is calculated on a radio photon by using a singularity free and collision free $\mathrm{N}$-body problem solution called dynamic universe model. Here the capabilities of this dynamic universe model are extended into micro world i.e. to light photons and radio wavelength photons and neutrinos etc. By doing so a real world very long baseline interferometry (VLBI) observations 
are explained [10]. The VLBI techniques give gravitational bending results in a wide range of values. Now dynamic universe model explains reason for such variation. The basic difference is that where present day physics considers gravitation effects of only sun or the main gravitating body only, the dynamic universe model considers gravitational effect of sun, planets, globular clusters, milky-way, local systems etc., and finds the universal gravitational force vector at that instant of time for that configuration of the Universe.

Can the gravitational effect of sun and moon be neglected on me when $i$ am standing on earth? No. For example, tide caused by sun and moon in oceans-- We observe high tide and low tide in the mornings and evenings, or on full-moon-day and no-moon-day. These tides are caused by gravitation of sun and moon only. So we can not neglect gravitation effect of sun and moon on earth. For better accuracies we have to consider planets also....

There is a large variation in the gravitational bending results of very long baseline interferometry (VLBI), in the field of radio astronomical observations of quasars, galaxies etc. This variation is clearly visible when the solar gravitational bending/deflection angle is plotted against solar elongation angle [11-14]

Astronomical jets observed from center of disk galaxies like milkyway: Many galaxies show the formation of astronomical jets from its center including our milkyway. These set of N-body simulations show the root cause of these formations.

\section{Observations that support dynamic universe model}

Mathematical prediction of existence of blue shifted galaxies: The forecasts of dynamic universe model in 2004 came true observationally in 2009 [8]. Dynamic universe model calculated to anticipate the existence of a large number of blue shifted galaxies which are much more higher than the 40 numbers known from the time of Astronomer Hubble in 1930s. It was confirmed by Hubble space telescope (HST) observations in the year 2009. Today the HST observed more than 10000 directly. In addition one can safely assume and prove various other types of galaxies such as Quasars, UV galaxies, X-ray, $\gamma$ - Ray sources and other blue galaxies etc., are also blue shifted galaxies. That means about $31.7 \%$ of galaxy count are blue shifted. One should not neglect such large number of blue shifted galaxies. It appears it is a Godly devotion to Bigbang cosmologies! The author submitted a paper to PRD in 2004.

SN1987A-Neutrino emission: Many flavours of Neutrinos are generated from sun and stars. One will find other flavours are generated from SN1987A. This covers the whole spectrum of Neutrinos. There are no unkown additional flavours generated from Bigbang.

\section{Other cosmologies-comparison}

Newton's static universe model requires fine balancing of bodies in all directions, so that all bodies stay in static equilibrium of attraction forces. This was described as such equilibrium as though a set of needles is finely balancing on their noses, any small disturbance will cause all to fall. Here in our dynamic universe model, gravitational attraction forces are balanced, by centrifugal forces not by balancing attraction forces. SITA proves that bodies will not collapse but revolve about each other. Dynamic universe model will not have Big-bang singularity, as we are proposing a nonexpanding anisotropic and heterogeneous universe model without considering the general relativity. This is a dynamic universe model without space-time continuum. No Big bang singularity. Hence singularity theorem is not applicable here. Hawking and Penrose in their singularity theorem said that 'Isotropic and homogeneous expanding universe, there must be a Big bang singularity some time in the past according to general theory of relativity. PCP was not considered true here as in steady state universe we need not assume any homogeneity and isotropy here at any point of time. This is a non expanding universe and matter need not be created to keep the density constant. The steady state cosmological model was presented by Hoyle The perfect cosmological principle (PCP) stated by Hoyle is that, Isotropy and homogeneity and other statistical properties of the universe are time independent. Universe has no beginning. No starting point for time scale. Matter is required to be created to keep the density constant in the expanding universe. $\{$ In a recent paper Aguirre and Gratton time like geodesic are not complete in Hoyle's Steady-state model. They proposed a geodesically complete Steady-state model, in which two universes are simultaneously present. In one of them, the universe is expanding and time is moving forwards, and in the other, it is contracting and time is moving backwards\}. Friedmann-RobertsonWalker models are popular. These are standard Bigbang models. Naturally all the problems inherent in the Bigbang models are present here also. In the absence of other working cosmological models, many workers choose these next. Missing mass, lesser age of the universe, anisotropy of cosmic microwave background, Bigbang singularity etc., are some of the problems present in these models. Bowen and Ferreira said, In models by de Sitter or any other matter filled models, there will be mass loss by scalar charges in these types of expanding universe models. That means a point like particle carrying charge $\mathrm{q}$, acts like a source for mass less scalar field. It looses its mass in time. There is one more popular line of thought, which is being seen now a days. They are cyclic universe models presented by many workers. We will see some the recent work done by Steinhardt and Turok, in which the universe starts from Bigbang to end up in Big-crunch only to start again in Bigbang to start the cycle. They attempted to say a little about, what happened before Bigbang. Hawking and Penrose for detailed work Hawking and Ellis in their singularity theorem, showed that Bigcrunch heads towards a cosmic singularity, where General relativity fails. After big crunch what happens, nobody knows. There is a basic problem in all these models, including String theory and M-theory; the matter density is significantly low, which makes these models impractical. In these models the universe is flat but not closed. So the question comes what happens to all these radiation? Steinhardt and Turok, presented another model of cyclic universe, to overcome the problem of failure of general relativity after Big-Crunch. They pushed the Big-crunch singularity into $5^{\text {th }}$ dimension, so that other three spatial and one time coordinates will be intact. It may be Steady state model or cyclic universe model; one thing is there in common. Both types of models ask for the creation of matter from vacuum. Earlier on this point the Bigbang people were criticizing the Steady state people. Now let's see about rotation models presented various authors from Gödel to Korotky and Obukhov.There were many authors. Gödel metric described the solution of General relativity with homogeneous spacetime and with casualty condition violated. All these people gave mainly a line element as a solution to Einstein's General relativity and tested that solution. Nobody talked about revolution. Mainly they argued about the rotation of universe, saying "when everything rotating, why not universe also?" But they have not considered the revolution of parts of the universe. Another difficulty faced by Korotky and Obukhov, is that it is impossible to combine pure rotation with expansion of universe in a solution of general relativity for a pure simple source. There were many authors who faced problems like closed time like curves (CTCs) Obukhov Yu N and Saulo Carneiro. The problems like non linearity of 
coordinate axes and interdependency between coordinate axes is still present inherently in all these models.

There is a fundamental difference between galaxie/systems of galaxies and systems that normally use statistical mechanics, such as molecules in a box. The molecules repel each other. But in gravitation we have not yet experienced any repulsive forces. Binny and Tremaine only attraction forces were seen. Einstein introduced cosmological constant $\lambda$ to introduce repulsive forces at large scales like inter galactic distances in his General relativity based cosmological considerations in for expanding universe 1917. This was not liked by many, and created turbulence in the scientific world. One of the reasons for his cosmological constant $\lambda$ is that he disliked the picture at infinity given by Newtonian gravitation. Though his ideas about infinity were good, the cosmological constant $\lambda$ and repulsive forces created havoc in the scientific community for at least last hundred years! Almost every worker/scientist in this field faced problems either conceptually or mathematically. Singularities were big hurdles for many of us.

Here Blue and Red shifted galaxies will be present simultaneously. We need not introduce large correction factors to convert Blue shifted galaxies into red shifted galaxies [15-20].

\section{Dynamic Universe Model: Evidences}

Presence of blue shifted galaxies in the universe, is the main evidence. Hubble deep space houses thousands of blue shifted galaxies which is one of the greatest mysteries for expanding universe models could not explain.

Our galaxy the Milky way is moving with a speed $454 \pm 125 \mathrm{~km} / \mathrm{sec}$ towards $\mathrm{l}=63 \pm 15^{\circ}$ and $\mathrm{b}=-11 \pm 14^{\circ}$ relative to distant part of samples and $474 \pm 164 \mathrm{~km} / \mathrm{sec}$ towards $\mathrm{l}=167 \pm 20^{\circ}$ and $\mathrm{b}=5 \pm 20^{\circ}$ relative to nearer part of samples. The local group comprising of Milky way, NGC6822, Andromida galaxy and other dwarf elliptical galaxies, Magellanic clouds rotate about their centers and revolve around a common center. SM Faber and David Burstain in their paper "Motions of galaxies in the neighborhood of Local group '\{presented in a symposium,' Large scale motions of universe' Princeton described the streeming motions towards the Great Attractor (located at $\mathrm{l}=309$ and $\mathrm{b}=+18$ ) by the local group, Virgo cluster, Ursa major, Centaurus, Camelopardalis, PerseusPisces etc ., clusters with speeds ranging up to $1000 \mathrm{~km} / \mathrm{sec}$. Please note the difference in directions of movement as well as speeds. All these clusters form a super cluster which also rotate and revolve about each other. Groups of super clusters form Filament structures and to grate walls and so on. This is how our universe is Lumpy and anisotropic even at large scale.

Another piece of supporting evidence for the dynamic universe model was there. There is a considerable discussion was as to whether GA: the Great attractor exists at all. For example Mathewson, Ford and Buckhorn have measured the peculiar velocities 1355 spiral galaxies. They find no backside in fall into GA region, rather a bulk flow of about $400 \mathrm{~km} / \mathrm{sec}$ on the scales of 100 ho- 1 MPC. Thus there is a considerable doubt about the existing of an attracting mass there. Both the parties find streaming motions or bulk flow. If there is no attracting mass, then why they are moving? this super cluster must be in revolution motion. Birch has discovered the asymmetric distribution of the angles of rotation of polarization vectors of 132 radio sources and tried to explain this via the global rotation. We think that the asymmetric distribution of the angles of rotation of polarization vectors is due to the galaxies or parts of clusters revolving in different directions.

\section{Conclusions}

Astronomical jets emerging from galaxy center of milkyway or any other galaxy are simulated here using dynamic universe model in this paper. This $\mathrm{N}$ - body model consists of 133 bodies of point masses, viz., 90 Wolf-Rayet stars, Galaxy Center, 23 Globular Cluster groups, 16 Milkyway parts, Andromeda and Triangulum Galaxies, and a Test neutron the path which was traced.

Lots of theoretical experiments were conducted with many types of assumed initial conditions, which did not give expected bending results and were discarded. That means only in certain conditions astronomical jets are formed as shown in the results section.

In all these simulations presented in this paper the time step is 1 sec, sharp bending of neutron path either after 1030 or 1650 iterations, is visible in $\mathrm{x}$ and $\mathrm{y}$ axes graphs only, and $\mathrm{z}$ axis don't show any bending. Another important observation is either Galaxy core mass was increased or near star masses were reduced slightly. The directions of the neutron initial velocities are slightly different from the first simulation to the others.

Probably this may be too early to conclude from these N-body simulation experiments. So our initial proposition is correct. In some conditions as discussed above, these bodies produce such gravitational effects on the neutrons travelling in between these bodies, these neutrons will take such will take some paths which are perpendicular to the central plane of Galaxy near the Galaxy center. Though it is heavier for my purse, purchase of new computer became necessary to take the continual computational load $[21,22]$.

\section{List of Files Attached with This Paper}

1. "Vvtc EUREKA PERP rev Results 2002xGC Less NS $2 \mathrm{sec}$ Densemass.xls"

2. "Vvtc rev Results 2002xGC Less NS 2 sec Densemass.xls"

3. "Vvtc rev 2000 100xGC Less NS Densemass.xls"

4. "Vvtc rev xy BENT 2000 100xGC Less NS Densemass.xls"

5 "Vvtc rev 2000 Galaxy core Less Densemass.xls"

6. "vvtc Eureka perp results consolidation.xls"

7. "vvtc initial conditions consolidation.xls"

\section{Acknowledgement}

I thank Almighty Vak for continuously guiding this research. I thankfully appreciate the support given by my wife Savitri while working on this paper.

\section{References}

1. Schödel (2002) "A star in a 15.2-year orbit around the supermassive black hole at the centre of the Milky Way". Nature 419: 694-696.

2. Reynolds, Christopher S (2008) Bringing black holes into focus. Nature 455 $39-40$

3. Pallab Ghosh (2008) Black hole confirmed in Milky Way.

4. Ghez AM, Salim S, Hornstein SD, Tanner A, Lu JR, et al. (2005) Stellar Orbits around the Galactic Center Black Hole. The Astrophysical Journal 620: 744757.

5. Gupta SNP (2010) Dynamic Universe Model: A singularity-free N-body problem solution. VDM Publishing, Germany.

6. Gupta SNP (2011) Dynamic Universe Model: SITA singularity free software VDM Publishing, Germany.

7. Gupta SNP (2011) Dynamic Universe Model: SITA software simplified. VDM Publishing, Germany. 
Citation: Gupta SNP (2015) Explaining Formation of Astronomical Jets Using Dynamic Universe Model. J Astrophys Aerospace Technol 3: 119. doi:10.4172/2329-6542.1000119

Page 8 of 8

8. Gupta SNP (2011) Dynamic Universe Model: Blue Shifted Galaxies Prediction Published. LAP Lambert Academic Publishing, Germany.

9. Gupta SNP (2011) Introduction to Dynamic Universe Model. International Journal of Scientific Research and Reviews 2: 203-226.

10. Gupta SNP (2014) Dynamic Universe Model Explains the Variations of Gravitational Deflection Observations of Very-Long-Baseline Interferometry (VLBI). Applied Physics Research 6: 1-9.

11. Gupta SNP (2014 a) Dynamic Universe Model's Prediction "No Dark Matter" in the Universe Came True! Applied Physics Research 6: 8-18.

12. Gupta SNP (2012) Singularity free N-body simulations DUMOC No-dark matter. COSPAR 13

13. TOI (2013) Search for Dark Matter Comes Up Empty So Far. Times Of India.

14. Akerib DS (2014) First results from the LUX dark matter experiment at the Sanford Underground. Phys Rev Lett 112.

15. Akerib DS (2013) Technical results from the surface run of the LUX dark matter experiment. Astroparticle Physics 45: 34-43.
16. Times NY (2013) Dark-matter-experiment-has-found-nothing-scientists-sayproudly. New York Times.

17. Davis UC (2013) Large Underground Xenon at UC Davis. Physics.

18. Mortlock DJ, Warren SJ, Venemans BP, Patel M, Hewett PC, et al. (2011) A luminous quasar at a redshift of $z=7: 085$. Nature 474: 616 .

19. Willott C (1963) A Star-like Object with Large Red-shift. Nature 197: 1040.

20. Shapiro SS, Davis JL, Lebach DE, Gregory JS (2004) Measurement of the Solar Gravitational Deflection of RadioWaves using Geodetic Very-LongBaseline Interferometry Data, 1979-1999. Physical Review Letters 92.

21. Sovers OJ, Fanselow JL, Jacobs CS (1997) Astrometry and geodesy with radio interferometry: experiments, models, results. Rev Mod Phys 70: 1-83.

22. Fomalont E, Kopeikin S, Lanyi G, Benson J (2009) Progress in Measurements of the Gravitational Bending of Radio Waves Using the VLBA. Astrophys 699: $1-22$ 